\title{
Cefazolin Prophylaxis for Total Joint Arthroplasty: Obese Patients are Frequently Underdosed and at Increased Risk for Periprosthetic Joint Infection
}

Running Title: Cefazolin Dosing in TJA

\author{
Alexander J. Rondon, MD, MBA ${ }^{1}$ \\ Michael M. Kheir, MD ${ }^{2}$ \\ Timothy L. Tan, MD \\ Noam Shohat, $\mathrm{MD}^{1,3}$ \\ Max R. Greenky, MD ${ }^{1}$ \\ Javad Parvizi, MD, FRCS ${ }^{1}$
}

${ }^{1}$ The Rothman Institute at Thomas Jefferson University, Philadelphia, PA 19107

${ }^{2}$ Department of Orthopaedics at Indiana University, Indianapolis, IN 46202

${ }^{3}$ Sackler Faculty of Medicine, Tel Aviv University, Ramat Aviv, Israel

\author{
Corresponding Author: \\ Javad Parvizi, MD, FRCS \\ The Rothman Institute \\ $125 \mathrm{~S} 9^{\text {th }}$ St. Ste 1000 \\ Philadelphia, PA 19107 \\ P: 267-339-7813 \\ F: 215-503-5651 \\ parvj@aol.com
}

This is the author's manuscript of the article published in final edited form as:

Rondon, A. J., Kheir, M. M., Tan, T. L., Shohat, N., Greenky, M. R., \& Parvizi, J. (2018). Cefazolin Prophylaxis for Total Joint Arthroplasty: Obese Patients are Frequently Underdosed and at Increased Risk for Periprosthetic Joint Infection. The Journal of Arthroplasty. https://doi.org/10.1016/j.arth.2018.06.037 
1 Cefazolin Prophylaxis for Total Joint Arthroplasty: Obese Patients are Frequently Underdosed and at Increased Risk for Periprosthetic Joint Infection 


\section{ABSTRACT}

7 Background: One of the most effective prophylactic strategies against periprosthetic joint

8 infection (PJI) is administration of perioperative antibiotics. Many orthopaedic surgeons

9 are unaware of the weight-based dosing protocol for cefazolin. This study aimed to

10 elucidate what proportion of patients receiving cefazolin prophylaxis are underdosed and

11 whether this increases the risk for PJI.

12

13 Methods: A retrospective study of 17,393 primary total joint arthroplasties (TJA)

14 receiving cefazolin as perioperative prophylaxis from 2005-2017 was performed. Patients

15 were stratified into two groups (underdosed and adequately dosed) based on patient

16 weight and antibiotic dosage. Patients that developed PJI within 1-year following index

17 procedure were identified. A bivariate and multiple logistic regression analysis were

18 performed to control for potential confounders and identify risk factors for PJI.

19

20 Results: The majority of patients weighing greater than $120 \mathrm{~kg}(95.9 \%, 944 / 984)$ were

21 underdosed. Underdosed patients had a higher rate of PJI at 1-year compared with

22 adequately dosed patients ( $1.51 \%$ vs. $0.86 \%, \mathrm{p}=0.002)$. Patients weighing greater than

$23120 \mathrm{~kg}$ had higher 1-year PJI rate than patients weighing less than $120 \mathrm{~kg}(3.25 \% \mathrm{vs}$.

$240.83 \%, \mathrm{p}<0.001$ ). Patients who were underdosed (odds ratio (OR) 1.665, $\mathrm{p}=0.006$ ) with

25 greater comorbidities (OR 1.259, $\mathrm{p}<0.001)$ were more likely to develop PJI at 1-year.

26

27 Conclusion: Cefazolin underdosing is common, especially for patients weighing more

28 than $120 \mathrm{~kg}$. Our study reports that underdosed patients were more likely to develop PJI. 
29 Orthopaedic surgeons should pay attention to the weight-based dosing of antibiotics in

30 the perioperative period to avoid increasing risk for PJI.

31

32 Keywords: Total Joint Arthroplasty; Periprosthetic Joint Infection; Perioperative

33 Antibiotics; Obesity; Dosing; Risk

34 


\section{INTRODUCTION}

36 One of the most effective strategies for prevention of periprosthetic joint infection

37 (PJI) has been the administration of perioperative antibiotics. The presence of antibiotics

38 in the serum can eliminate the bacteria that gain access to the surgical site during total

39 joint arthroplasty (TJA) and in turn reduce the incidence of surgical site infection [1].

40 Current practice is to administer first or second generation cephalosporin to all patients

41 undergoing TJA, unless contraindicated [2]. Despite the widespread use of cefazolin as a

42 perioperative antibiotic for TJA patients, many surgeons are unaware of cefazolin's

43 weight-based dosing. Thus, the goals of the present study are (1) to ascertain what

44 proportion of TJA patients receiving cefazolin are adequately dosed and (2) if under-

45 dosing was associated with increased risk of subsequent PJI.

At our institution, the recommended dose of cefazolin has traditionally been 2

47 grams intravenously. Current guidelines for antimicrobial prophylaxis recommend

48 weight-based dosing protocols starting the cefazolin dose at 1 gram (g) if a patient weighs

49 less than 60 kilograms $(\mathrm{kg}), 2$ grams if patient weights between $60 \mathrm{~kg}$ and $120 \mathrm{~kg}$, and 3

50 grams if patient weight over $120 \mathrm{~kg}[2,3]$. A previous study at our institution found the

51 majority of patients receiving vancomycin as perioperative prophylaxis were underdosed

52 according to weight-based dosage recommendations (15 milligrams $/ \mathrm{kg}$ ) [4].

53 Given the increasing prevalence of obesity $[5,6]$ in the TJA population, many

54 patients may be inadequately dosed for antibiotics. Thus, the effective drug concentration

55 may not be met to provide bactericidal effects and subsequently may predispose patients

56 to an increased risk for PJI. We hypothesis patients who are underdosed are at increased

57 risk of adverse events and infection. 


\section{MATERIALS AND METHODS}

After Institutional Review Board approval, a retrospective review of 24,439

61 patients undergoing primary TJA at a single institution was performed from 2005-2017.

62 All patients with primary TJA, with record of the perioperative antibiotic and dosage

63 administrated were included in this study. Patients with aseptic revision TJA were

64 excluded. The perioperative antibiotic and dosage were then obtained for the patient

65 population, resulting in a cohort of 17,393 of patients receiving cefazolin as perioperative

66 prophylactic antibiotic. Patients who received other types of perioperative prophylactic

67 antibiotics (i.e. Vancomycin) other than Cefazolin were excluded from the study. Patients

68 with a history of prior infection in the same joint or unavailable antibiotic information

69 were excluded from the study. An electronic query and chart review was then performed

70 to identify demographic information, height, weight, body mass index (BMI), joint,

71 laterality, length of stay, operative time, time to incision from administration of cefazolin,

72 and Charlson comorbidities. Demographic information of the cohort is presented in Table 731.

74 Using the generalized dosing protocol of $1 \mathrm{~g}$ for patients weighing below $60 \mathrm{~kg}, 2$

$75 \mathrm{~g}$ for patients weighing between $60 \mathrm{~kg}$ and $120 \mathrm{~kg}$, and $3 \mathrm{~g}$ for patients weighing $120 \mathrm{~kg}$

76 or greater for cefazolin, proper dosage was calculated for each patient. These values were

77 then compared to the actual dose given to the patients at time of surgery. Patients were

78 assessed as either underdosed $(<1 \mathrm{~g}$, if patient weighed between $60 \mathrm{~kg}-120 \mathrm{~kg}$ and was

79 given $<2 \mathrm{~g}$, or if patient weighed $120 \mathrm{~kg}$ or more and was given $<3 \mathrm{~g}$ ) or adequately 
80 dosed (if appropriately dosed based on weight). Cefazolin was administered within 60

81 minutes of incision in all cases.

The cohort was then cross-referenced with an institutional PJI database to identify

83 patients with PJI. We defined PJI in patients based on the International Consensus

84 Meeting criteria[7]. A subsequent manual chart review was undertaken to verify PJI

85 outcomes and ensure the correct joint and laterality.

86 The primary endpoint was to assess the incidence of 1-year PJI following TJA in

87 patients who were underdosed versus adequately dosed.

88 Statistical Analysis

89 All statistical analyses were performed with PJI rate analyzed among the two

90 dosing groups and by weight class. Bivariate analyses were performed to compare

91 demographics, perioperative variables between the two dosing groups and weight class.

92 A multivariate logistic regression model was utilized to determine risk factors for PJI

93 based on the following: antibiotic dosing, dosing status, age, patient weight, BMI, gender,

94 joint, length of stay, and Charlson Comorbidity index. All statistical analyses were

95 performed using R 2.15.1 (R Foundation for Statistical Computing, Vienna, Austria) and

96 an alpha level of 0.05 was used to evaluate significance. All analyses were conducted

97 with Generalized estimating equations (GEE) to account for the clustering within patients

98 who had multiple admissions. The GEE specified a binary distribution with a logit link

99 for analyzing the dichotomous outcomes.

100

$101 \quad$ RESULTS 
All patients included in the study received cefazolin preoperatively within 60

103 minutes as the main antibiotic prophylaxis. Of the 984 patients weighing $120 \mathrm{~kg}$ or

104 greater, the majority were underdosed $(95.9 \%, 944 / 984)$. For patients weighing less than

$105120 \mathrm{~kg}$, most were adequately dosed $(88.3 \%, 14,497 / 16,409)$. Overall, $83.6 \%$

$106(14,537 / 17,393)$ of patients were adequately dosed for cefazolin prophylaxis. Of note,

$1070.10 \%(18 / 17,393)$ patients were overdosed, however, none developed PJI at 1-year and

108 all weighed between 60 and $120 \mathrm{~kg}$.

109 Among primary TJAs, underdosed patients had a higher rate of 1-year PJI

110 compared with adequately dosed patients ( $1.51 \%$ vs. $0.86 \%, \mathrm{p}=0.002)$. When stratified by

111 weight, patients weighing greater than or equal to $120 \mathrm{~kg}$ had higher 1-year PJI rate than

112 patients weighing less than $120 \mathrm{~kg}(3.25 \%$ vs. $0.83 \%, \mathrm{p}<0.001)$ (Table 1$)$.

113 Bivariate analysis demonstrated that patients who were underdosed (adjusted odds

114 ratio (OR) 1.762, $\mathrm{p}=0.002)$, male (OR 1.517, $\mathrm{p}=0.014)$, those with greater comorbidities

115 (OR 1.251, $\mathrm{p}<0.001)$, and higher weight (OR 1.025, $\mathrm{p}<0.001)$ were more likely to

116 develop PJI within 1-year (Table 2). Following multivariate regression analyses, these

117 trends remained significant with underdosed (OR 1.665, $\mathrm{p}=0.006)$ and patients with

118 greater comorbidities (OR 1.259, $\mathrm{p}<0.001$ ) having a higher rate of PJI at 1-year (Table 3).

120 DISCUSSION

121 The efficacy and value of perioperative antibiotics for surgical prophylaxis has

122 been proven in the literature [8]. Recent studies have supported current universal

123 antibiotic prophylaxis versus providing treatment based on individual comorbidities[9].

124 The most appropriate antibiotic therapy recommended for patients undergoing TJA is a 
125 first or second generation cephalosporin due to its broad spectrum of action, cost

126 effectiveness, and ability to cover both gram positive and gram negative organisms

$127[3,10,11]$. Furthermore, cephalosporins are bactericidal and have excellent distribution

128 profiles in synovium, muscle, hematomas, and bone [12]. The current American

129 Academy of Orthopaedic Surgeons (AAOS) guidelines recommend patients receive

130 prophylactic antibiotics within one hour prior to surgical incisions and be discontinued

131 within 24 hours following the end of surgery [13].

132 The literature has previously reported on the necessity for weight-based dosing of

133 perioperative antibiotics. While the current guidelines from the Center for Disease

134 Control and Prevention, World Health Organization, and National Institute for Healthcare

135 and Excellence do not provide dosing recommendation, the Society for Healthcare

136 Epidemiology of America and the International Consensus Meeting (ICM) on PJI

137 strongly agreed that preoperative antibiotics weight-based dosing is valid and warranted

$138[2,14,15,3,16]$. However, for adult patients, standard antibiotic dosing remains a common

139 practice as it is safe, effective, and conveniently avoids the need for calculations, thus

140 reducing the potential for medication errors[17]. Different ranges for perioperative

141 cefazolin dosing protocols has been reported from standard adult dose of $2 \mathrm{~g}$ [18] to

142 weight-based dosing of $1 \mathrm{~g}$ for patients weighing less than $80 \mathrm{~kg}$ or $2 \mathrm{~g}$ for patients

143 weighing greater than $80 \mathrm{~kg}$ [19]. The American Society of Health-System Pharmacists

144 (ASHP) recommends a weight-based protocol of $1 \mathrm{~g}$ from patients weighing less than 60

$145 \mathrm{~kg}, 2 \mathrm{~g}$ for patients weighing $60-120 \mathrm{~kg}$, and $3 \mathrm{~g}$ for patients weighing $120 \mathrm{~kg}$ or more

146 [3]. Our institution follows these weight-based guidelines utilizing both $60 \mathrm{~kg}$ and $120 \mathrm{~kg}$

147 cutoffs, however, as illustrated by the results of the present study, the majority of patient 
148 weighing above $120 \mathrm{~kg}$ were underdosed by recieving $2 \mathrm{~g}$ of antibiotics. Similar to our

$1495.7 \%$ rate of patients weighing greater than $120 \mathrm{~kg}$, the prevalence of extreme obesity

150 (BMI > 40) in the United States has been reported at 7.7\% [5,20]. When assuming

151 estimates of 1,000,000 TJA performed annually and an underdosing rate of greater than

$15290 \%$ for the extreme obese population, fifty thousand TJA patients are likely underdosed

153 each year [21]. Given the significant rise in obesity and morbid obesity, increased

154 scrutiny with respect to perioperative antibiotic prophylaxis is warranted to ensure that

155 this population is not underdosed [5,6].

156 The literature has previously reported on factors affecting the dosing of

157 perioperative antibiotics, specifically patient weight. One study demonstrated that $2 \mathrm{~g}$ of

158 cefazolin provided 5 hours of adequate levels of prophylactic protection for patients

159 regardless of their BMI [18]. Edminston et al. reported on cefazolin serum concentrations

160 in morbidly obese undergoing gastric bypass, concluding that $2 \mathrm{~g}$ of cefazolin may not be

161 sufficient for patients with a BMI of $50 \mathrm{Kg} / \mathrm{m}^{2}$ or greater [22]. A prospective randomized

162 controlled trial (RCT) of morbidly obese patients undergoing gastroplasty reported

163 decreased wound infection rate from $16.5 \%$ to $5.6 \%$ when cefazolin dosed was increased

164 from $1 \mathrm{~g}$ to $2 \mathrm{~g}$ [23]. In contrast to our study, Kheir et al. found a comparable PJI rate

165 among stratified vancomycin dosage groups (underdosed $2 \%$, adequately dosed $2 \%$,

166 overdosed $2 \%, \mathrm{p}=0.995)$, however reported that $64 \%$ of patients receiving vancomycin as

167 prophylaxis were underdosed and overall patients receiving vancomycin prophylaxis

168 were at an increased risk of PJI (OR 1.587, $\mathrm{p}=0.048)$ compared to patients receiving

169 cefazolin prophylaxis [4]. Sharareh et al. found no difference in cefazolin concentration

170 in trabecular bone with respect to patient weight [24]. Additionally, Manrique et al. 
reported that patients undergoing total knee arthroplasty (TKA) who were underweight had a higher likelihood of surgical site infection compared to other weight groups [25]. However, we do recognize that the majority of these studies report results by BMI as opposed to weight, which may create confusion as dosing is based on weight not BMI. The present study reports data by weight category as opposed to BMI.

The present study has several limitations. First, the retrospective nature of the study is subject to the inherent bias of retrospective work. Second, underdosed patients weighing greater than $120 \mathrm{~kg}$ may have been predisposed to adverse conditions due to morbidity associated with obesity rather than inadequate dosing of cefazolin. Third, despite having more than 17,000 patients, we may still be underpowered given the low rate of PJI. Fourth, the present study encompasses a large time-period and there may be protocol changes over this time-period that may not be accounted for. Fifth, while our study primarily focuses on weight, other factors that influence antibiotic dosing such as liver and kidney function, gender, and fat distribution were not considered. However, despite the aforementioned limitations, the present study does bring to light important dosing considerations when treating patients weighing $120 \mathrm{~kg}$ or more.

Perioperative antibiotics remain an important strategy in protection against PJI, one of the most devastating complications following TJA. While the majority of patients remain adequately dosed, underdosing of cefazolin in the obese patient is common. We suggest orthopaedic surgeons incorporate proper weight based antibiotic dosing in their preoperative planning. Orthopaedic surgeons must be vigilant when treating patients weighing $120 \mathrm{~kg}$ or greater as failure to adequately dose their perioperative antibiotics can unnecessarily predispose this population to PJI. 
198 [1] Alijanipour P, Heller S, Parvizi J. Prevention of periprosthetic joint infection: 199 what are the effective strategies? J Knee Surg 2014;27:251-8. doi:10.1055/s-00342001376332.

201 [2] Hansen E, Belden K, Silibovsky R, Vogt M, Arnold WV, Bicanic G, et al. 202 Perioperative antibiotics. J Arthroplasty 2014;29:29-48. doi:10.1016/j.arth.2013.09.030.

203 [3] Bratzler DW, Dellinger EP, Olsen KM, Perl TM, Auwaerter PG, Bolon MK, et al. 204 Clinical practice guidelines for antimicrobial prophylaxis in surgery. Surg Infect 205 2013;14:73-156. doi:10.1089/sur.2013.9999.

206 [4] Kheir MM, Tan TL, Azboy I, Tan DD, Parvizi J. Vancomycin Prophylaxis for 207 Total Joint Arthroplasty: Incorrectly Dosed and Has a Higher Rate of Periprosthetic 208 Infection Than Cefazolin. Clin Orthop 2017;475:1767-74. doi:10.1007/s11999-017209 5302-0.

210 [5] Flegal KM. Epidemiologic aspects of overweight and obesity in the United States. 211 Physiol Behav 2005;86:599-602. doi:10.1016/j.physbeh.2005.08.050.

212 [6] Pai MP, Bearden DT. Antimicrobial Dosing Considerations in Obese Adult 213 Patients. Pharmacother J Hum Pharmacol Drug Ther 2007;27:1081-91.

214 doi:10.1592/phco.27.8.1081.

215 [7] Zmistowski B, Della Valle C, Bauer TW, Malizos KN, Alavi A, Bedair H, et al.

216 Diagnosis of periprosthetic joint infection. J Arthroplasty 2014;29:77-83.

217 doi:10.1016/j.arth.2013.09.040.

218 [8] AlBuhairan B, Hind D, Hutchinson A. Antibiotic prophylaxis for wound

219 infections in total joint arthroplasty: a systematic review. J Bone Joint Surg Br

220 2008;90:915-9. doi:10.1302/0301-620X.90B7.20498.

221 [9] Tan TL, Gomez MM, Kheir MM, Maltenfort MG, Chen AF. Should Preoperative 222 Antibiotics Be Tailored According to Patient's Comorbidities and Susceptibility to 223 Organisms? J Arthroplasty 2017;32:1089-1094.e3. doi:10.1016/j.arth.2016.11.021.

224 [10] Hansen E, Belden K, Silibovsky R, Vogt M, Arnold W, Bicanic G, et al.

225 Perioperative antibiotics. J Orthop Res Off Publ Orthop Res Soc 2014;32 Suppl 1:S31-

226 59. doi:10.1002/jor.22549.

227 [11] Bratzler DW, Houck PM, Surgical Infection Prevention Guideline Writers

228 Workgroup. Antimicrobial prophylaxis for surgery: an advisory statement from the

229 National Surgical Infection Prevention Project. Am J Surg 2005;189:395-404.

230 doi:10.1016/j.amjsurg.2005.01.015.

231 [12] Neu HC. Cephalosporin antibiotics as applied in surgery of bones and joints. Clin 232 Orthop 1984:50-64.

233 [13] American Academy of Orthopaedic Surgeons; Information statement:

234 recommendations for the use of intravenous antibiotic prophylaxis in primary total joint 235 arthroplasty. AAOS n.d.

236 [14] WHO | Global guidelines on the prevention of surgical site infection. WHO n.d. 237 http://www.who.int/gpsc/ssi-prevention-guidelines/en/ (accessed November 19, 2017).

238 [15] Leaper D, Burman-Roy S, Palanca A, Cullen K, Worster D, Gautam-Aitken E, et 239 al. Prevention and treatment of surgical site infection: summary of NICE guidance. BMJ $240 \quad$ 2008;337:a1924. 
241 [16] Guidelines Library | Infection Control | CDC n.d.

242 https://www.cdc.gov/infectioncontrol/guidelines/index.html (accessed November 19,

243 2017).

244 [17] Pan S-D, Zhu L-L, Chen M, Xia P, Zhou Q. Weight-based dosing in medication

245 use: what should we know? Patient Prefer Adherence 2016;10:549-60.

246 doi:10.2147/PPA.S103156.

247 [18] Ho VP, Nicolau DP, Dakin GF, Pomp A, Rich BS, Towe CW, et al. Cefazolin

248 dosing for surgical prophylaxis in morbidly obese patients. Surg Infect 2012;13:33-7.

249 doi:10.1089/sur.2010.097.

250 [19] Meehan J, Jamali AA, Nguyen H. Prophylactic antibiotics in hip and knee

251 arthroplasty. J Bone Joint Surg Am 2009;91:2480-90. doi:10.2106/JBJS.H.01219.

252 [20] Flegal KM, Kruszon-Moran D, Carroll MD, Fryar CD, Ogden CL. Trends in

253 Obesity Among Adults in the United States, 2005 to 2014. JAMA 2016;315:2284-91.

254 doi:10.1001/jama.2016.6458.

255 [21] Kurtz S, Ong K, Lau E, Mowat F, Halpern M. Projections of primary and revision 256 hip and knee arthroplasty in the United States from 2005 to 2030. J Bone Joint Surg Am 257 2007;89:780-5. doi:10.2106/JBJS.F.00222.

258 [22] Edmiston CE, Krepel C, Kelly H, Larson J, Andris D, Hennen C, et al.

259 Perioperative antibiotic prophylaxis in the gastric bypass patient: do we achieve

260 therapeutic levels? Surgery 2004;136:738-47. doi:10.1016/j.surg.2004.06.022.

261 [23] Forse RA, Karam B, MacLean LD, Christou NV. Antibiotic prophylaxis for

262 surgery in morbidly obese patients. Surgery 1989;106:750-6; discussion 756-757.

263 [24] Sharareh B, Sutherland C, Pourmand D, Molina N, Nicolau DP, Schwarzkopf R.

264 Effect of Body Weight on Cefazolin and Vancomycin Trabecular Bone Concentrations in

265 Patients Undergoing Total Joint Arthroplasty. Surg Infect 2016;17:71-7.

266 doi:10.1089/sur.2015.067.

267 [25] Manrique J, Chen AF, Gomez MM, Maltenfort MG, Hozack WJ. Surgical site

268 infection and transfusion rates are higher in underweight total knee arthroplasty patients.

269 Arthroplasty Today 2017;3:57-60. doi:10.1016/j.artd.2016.03.005.

270

271 
Table 1: Demographic information and dosing information

\begin{tabular}{|c|c|c|c|}
\hline \multicolumn{4}{|c|}{ Cohort $(n=17,393)$} \\
\hline & $\begin{array}{c}\text { Adequately } \\
\text { Dosed } \\
(n=\mathbf{1 4 , 5 3 7})\end{array}$ & $\begin{array}{c}\text { Underdosed } \\
(\mathbf{n}=\mathbf{2 , 8 5 6})\end{array}$ & P value \\
\hline Age (year) & $63.5(0.09)$ & $63.5(0.22)$ & 0.849 \\
\hline Gender (male) & $6417(44.1 \%)$ & $1736(60.8 \%)$ & $<0.001$ \\
\hline BMI & $29.55(0.04)$ & $32.11(0.13)$ & $<0.001$ \\
\hline Weight (kg) & $84.41(0.14)$ & $97.17(0.49)$ & $<0.001$ \\
\hline CCI & $0.391(0.01)$ & $0.386(0.02)$ & $<0.001$ \\
\hline Joint (knee) & $6895(47.4 \%)$ & $1320(46.2 \%)$ & 0.235 \\
\hline LOS & $2.67(0.02)$ & $3.05(0.05)$ & $<0.001$ \\
\hline 90 day Readmission & $555(3.8 \%)$ & $128(4.5 \%)$ & 0.113 \\
\hline 1-year PJI & $125(0.86 \%)$ & $43(1.51 \%)$ & 0.002 \\
\hline \multicolumn{4}{|c|}{ Stratified by Weight } \\
\hline & $\begin{array}{c}<120 \mathrm{~kg} \\
(\mathrm{n}=16,409)\end{array}$ & $\begin{array}{l}\geq 120 \mathrm{~kg} \\
(\mathrm{n}=984)\end{array}$ & P value \\
\hline Age (year) & $63.9(0.09)$ & $57.8(0.29)$ & 0.039 \\
\hline CCI & $0.387(0.01)$ & $0.441(0.03)$ & 0.100 \\
\hline Joint (knee) & $8047(46.6 \%)$ & $567(57.8 \%)$ & $<0.001$ \\
\hline LOS & $2.71(0.02)$ & $3.11(0.08)$ & 0.618 \\
\hline 90 day Readmission & $614(3.7 \%)$ & $69(7.0 \%)$ & $<0.001$ \\
\hline Underdosed & $1912(11.7 \%)$ & $944(95.9 \%)$ & $<0.001$ \\
\hline 1-year PJI & $136(0.83 \%)$ & $32(3.25 \%)$ & $<0.001$ \\
\hline
\end{tabular}

Data presented in table as mean (standard error) or number (percentage)

Abbreviations: BMI, Body Mass Index; kg, kilogram; CCI, Charlson Comorbidity index; LOS, Length of Stay; PJI, Periprosthetic Joint Infection 
Table 2: Bivariate analysis of Cefazolin and 1-year PJI

\begin{tabular}{|l|r|r|}
\hline & \multicolumn{1}{|l|}{$\begin{array}{l}\text { Adjusted } \\
\text { Odds } \\
\text { Ratio }\end{array}$} & P Value \\
\hline Underdosed & 1.762 & 0.002 \\
\hline Gender (male) & 1.517 & 0.014 \\
\hline Joint (knee) & 0.900 & 0.512 \\
\hline Younger Age (year) & 1.010 & 0.174 \\
\hline Weight (kg) & 1.025 & $<0.001$ \\
\hline BMI & 1.080 & $<0.001$ \\
\hline CCI & 1.251 & $<0.001$ \\
\hline
\end{tabular}

Abbreviations: PJI, Periprosthetic Joint Infection; kg, kilogram; BMI, Body Mass Index; CCI, Charlson Comorbidity index 
Table 3: Multivariate analysis for weight-based dosing of Cefazolin and likelihood of PJI

\begin{tabular}{|l|r|r|}
\hline \multicolumn{3}{|c|}{ Regression } \\
\hline & $\begin{array}{c}\text { Adjusted } \\
\text { Odds Ratio }\end{array}$ & $\boldsymbol{P}$ Value \\
\hline Underdosed & 1.665 & 0.006 \\
\hline Gender (male) & 1.372 & 0.067 \\
\hline Younger Age (year) & 1.011 & 0.130 \\
\hline CCI & 1.259 & $<0.001$ \\
\hline
\end{tabular}

Abbreviations: PJI, Periprosthetic Joint Infection; kg, kilogram; CCI, Charlson Comorbidity index 